\title{
Experimental Investigation On EDM Processes By Tool Rotating Speed
}

\author{
Nimo Singh Khundrakpam, Gurinder Singh Brar and Dharmpal Deepak
}

\begin{abstract}
Electrical Discharge Machine is commonly used non-traditional machining process to machine high strength advance materials which are difficult to machine. The present research investigates the effect of tool rotating speed to the responses viz. MRR, TWR and surface roughness (Ra) of Electric Discharge Machine (EDM) processes viz. EDM, Dry-EDM, Near Dry EDM which used the liquid, gas and liquid-gas dielectric respectively. It is found that the increased in tool rotating speed increases MRR then decreases. It is also found that increase in tool rotating speed decreases Surface roughness (Ra). TWR for Dry-EDM and NDEDM compared to Wet EDM is negligible..
\end{abstract}

Keywords--- EDM, Dry EDM, ND-EDM, Tool Rotation Speed, MRR, TWR, Ra

\section{INTRODUCTION}

$\mathrm{E}$ lectrical discharge machining (EDM) is a commonly used non-traditional machining process to produce dies, molds, automotive industry and also surgical components [1]. It was first developed in the late 1940s [2] where material removal is done by series of electrical discharges between tool and the work piece within a dielectric medium [3]. EDM does not make any direct contact between the electrode and the work piece that can eliminate mechanical stresses and vibration problems. In EDM process, electrical energy is converted into thermal energy that creates plasma channel of temperature 8000$12000^{\circ} \mathrm{C}$ between the tool and workpiece which is sufficient to melt the workpiece and the tool. The plasma channel spreads and causes and jumping out of the molten materials are jumping out from the workpiece and tool surfaces when a sudden drop in temperature by cutting off the pulse energy. The erosive effect of the electrical discharges is the key role to remove material from the

Nimo Singh Khundrakpam, PhD. Research Scholar, Department of Mechanical Engineering, IKG Punjab Technology University, Kapurthala, India. E-mail:nimspn@gmail.com

Gurinder Singh Brar, Assistant Professor, Department of Mechanical Engineering, GNDEC, Ludhiana, India. E-mail: brar.gurinder@gmail.com

Dharmpal Deepak, Assistant Professor, Department of Mechanical Engineering, Punjabi University, Patiala, India. E-mail: deepakbass@yahoo.com DOI:10.9756/BIJIEMS.8309 workpiece [4]. EDM process has limitation such as low MRR, high surface roughness, high TWR, formation of recast layer on workpiece surfaces and environmental hazards. To overcome the limitation of EDM, new processes like dry EDM and near-dry EDM have been developed.

In dry EDM, high-pressure air or gas such as air, oxygen, helium, nitrogen, and argon is supplied through the hollow tool electrode as a dielectric fluid [5]. The gas is injected inside the tool electrode with high pressure, which removes melted material from the working gap and cools down the tool electrode and the workpiece.

The role of the gas is to withstand as insulator and remove the debris between tool and workpiece. It also use as a coolant for tool and workpiece. However, low stability of arc column as compared to wet EDM is the Dry EDM process limitation [6]. First investigation of ND-EDM was carried out by Tambura et al. in 1989 [7]. The near-dry EDM process used a liquid dielectric is replaced by a mixture of gas and liquid dielectric. In 2007, Kao et al. was carried a meeningful investigation to study the effects of the electrode material and dielectric medium for roughing and finishing operations after first investigation. The dielectric is flushing through the hollow tool at high speed on the workpiece surface [8].

In the present investigation, effect of tool rotating speed to the responses viz. MRR, TWR and Ra of Wet EDM, Dry EDM and ND-EDM were studied.

\begin{tabular}{|ll|}
\hline NOMENCLATURE \\
$\mathrm{N}$ & Tool Rotating Speed $(\mathrm{rpm})$ \\
MRR & Material Removal Rate $\left(\mathrm{mm}^{3} / \mathrm{min}\right)$ \\
TWR & Tool Wear Rate $\left(\mathrm{mm}^{3} / \mathrm{min}\right)$ \\
$\mathrm{Ra}$ & Arithmetical mean surface roughness $(\mu \mathrm{m})$ \\
\hline
\end{tabular}

\section{EXPERIMENTAL SETUP}

The experiment was investigate using Savita ZNC EDM having tool rotating attachments. An air compressor is used to supply compressed air between the electrodes gap for Dry EDM. The Near dry minimal quantity lubrication (MQL) device is used to develop two phase liquid and gas mixture for the ND-EDM. Experiment was conducted by using copper as electrode and EN-8 as 
workpiece. Surface roughness value $\mathrm{Ra}(\mu \mathrm{m})$ is measured by using Mitutoyo Sutronic S-128. MRR and TWR of EDM process was calculated by weight loss method as shown in equations (1) \& (2) respectively.

$$
\begin{gathered}
M R R=\frac{\left[W_{i}-W_{f}\right]}{\rho_{w} T} \times 1000 \mathrm{~mm}^{3} / \mathrm{min} \\
T W R=\frac{\left[T_{i}-T_{f}\right]}{\rho_{t} T} \times 1000 \mathrm{~mm}^{3} / \mathrm{min}
\end{gathered}
$$

Where,

$\mathrm{W}_{\mathrm{i}}$ and $\mathrm{W}_{\mathrm{f}}$ are the initial and final weight of workpiece in $g m, T_{i}$ and $T_{f}$ are the initial and final weight of tool in gm, $\rho_{\mathrm{w}}$ and $\rho_{\mathrm{t}}$ are the density of workpiece and tool in $\mathrm{gm} / \mathrm{cm}^{3}$ and $\mathrm{T}$ is the machining time in mins.

The chemical properties of EN-8 steel workpiece and copper tool is shown in Table 1. The air is mixed with the EDM oil at the MQL system and used as dielectric medium for the ND-EDM.

Table 1: Chemical properties of copper and EN-8 Steel

\begin{tabular}{lll}
\hline & EN-8 Steel & Copper \\
\hline \multirow{3}{*}{ Composition } & $(\mathrm{C}+\mathrm{Si}+\mathrm{Mn}+\mathrm{S}+\mathrm{P})=$ & \\
& $0.40+0.25+0.80+0.05$ & Copper $(99 \%)$ \\
Hardness & +0.05 & \\
Density & $255 \mathrm{BHN}$ & $40 \mathrm{BHN}$ \\
Melting Point & $7.8 \mathrm{~g} / \mathrm{cm} 3$ & $8.90 \mathrm{~g} / \mathrm{cm}^{3}$ \\
\hline
\end{tabular}

Levels for various process parameters and their selected level are shown in Table 2 The selected response variables for this study are the MRR and TWR.

Table 2: Levels for various control parameters

\begin{tabular}{lll}
\hline Parameter & Units & No of Levels \\
\hline Polarity (P) & --- & Straight \\
Discharge Current (Id) & A & 4 \\
Gas Flow Pressure (GFP) & Bar & 6 \\
Pulse on Time (Ton) & $\mu \mathrm{s}$ & 250 \\
Gap Voltage (V) & V & 4 \\
Machining Time & min & 10 \\
Tool Rotating Speed (N) & RPM & $0,500,1000,1500$, \\
\hline
\end{tabular}

\section{RESULT AND ANALYSIS}

The three-experimental values were taken for each experimental run and average of them were find out. The average response table for MRR, TWR and Ra for Wet EDM, Dry EDM and ND-EDM process is tabulated in the Table 3.

\section{A. Analysis of MRR}

The effect of tool rotating speed to MRR is shown in Figure 1. It was found that MRR increased slightly then decreased rapidly with the increase of tool rotating speed. This can be occurred due to the effect of interruption on discharge energy by tool rotation for all EDM processes. ND-EDM has higher MRR compared to other EDM process. All the processes have like value of MRR for higher tool rotating speed (nearly at $2000 \mathrm{rpm}$ ). Maximum MRR of ND-EDM was found in range of 800$900 \mathrm{rpm}$ of tool rotation speed.

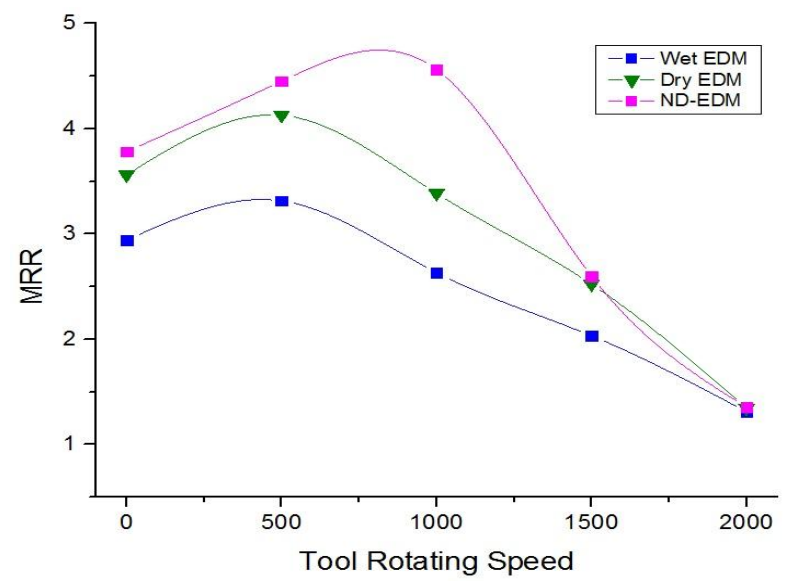

Figure 1: Effect of Tool Rotating Speed vs MRR

\section{B. Analysis of TWR}

The effect of the tool rotating speed to the TWR is shown in Figure 2. It was noted that the increased in the tool rotating speed has no effect on the TWR for NDEDM and Dry EDM. This can be occurred due to the effect of the pressurized gas to cool down the tool electrode and the workpiece. TWR of wet EDM increased with increase of the tool rotating speed. So, Dry-EDM and ND-EDM is mostly preferable for the mass production due to negligible tool wear.

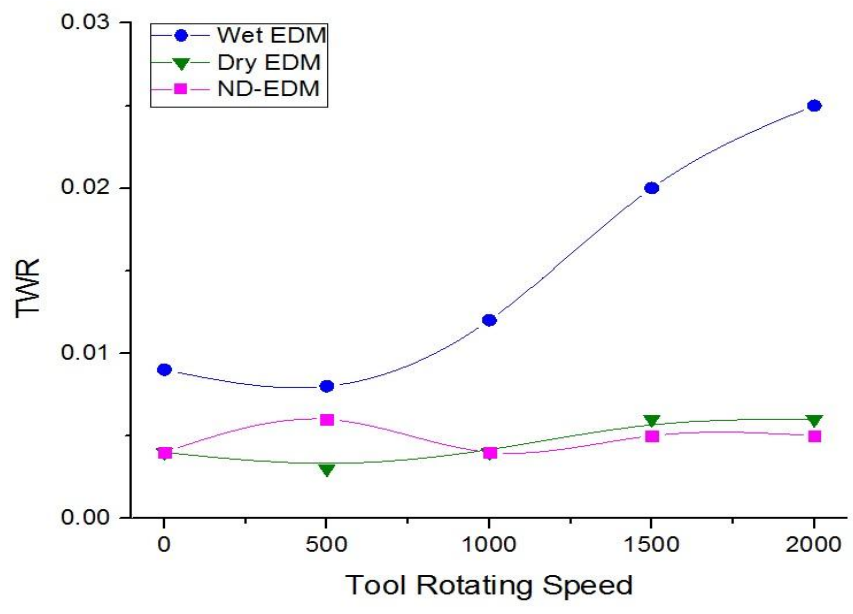

Figure 2: Effect of Tool Rotating Speed vs TWR 
Table 3: Response table for EDM, Dry EDM and ND-EDM process

\begin{tabular}{cllllllllll}
\hline & \multicolumn{3}{l}{ Wet EDM } & \multicolumn{3}{c}{ Dry EDM } & & \multicolumn{2}{c}{ ND-EDM } \\
Run & $\begin{array}{l}\text { Tool } \\
\text { Rotating } \\
\text { Speed }\end{array}$ & MRR & TWR & Ra & MRR & TWR & Ra & MRR & TWR & Ra \\
\hline 1 & 0 & 2.941 & 0.009 & 7.264 & 3.562 & 0.004 & 6.846 & 3.782 & 0.004 & 6.341 \\
2 & 500 & 3.315 & 0.008 & 7.02 & 4.128 & 0.003 & 6.677 & 4.452 & 0.006 & 6.272 \\
3 & 1000 & 2.632 & 0.012 & 6.195 & 3.386 & 0.004 & 5.866 & 4.562 & 0.004 & 5.567 \\
4 & 1500 & 2.033 & 0.02 & 5.59 & 2.523 & 0.006 & 5.371 & 2.598 & 0.005 & 5.486 \\
5 & 2000 & 1.311 & 0.025 & 5.22 & 1.345 & 0.006 & 5.148 & 1.354 & 0.005 & 5.026 \\
\hline
\end{tabular}

C. Analysis of $R a$

The effect of tool rotating speed to the surface roughness is shown in Figure 3. It can be concluded from the Figure 3 that Surface roughness ( $\mathrm{Ra}$ ) decreased with increased of the tool rotating speed. Surface the tool rotating speed. Surface roughness Ra value of Wet EDM, Dry EDM and ND-EDM were closed at the higher value of tool rotating speed. This can be occurred due to the effect of the pressurized gas which helps to remove melted material from the working gap and cools down the tool electrode and the workpiece.

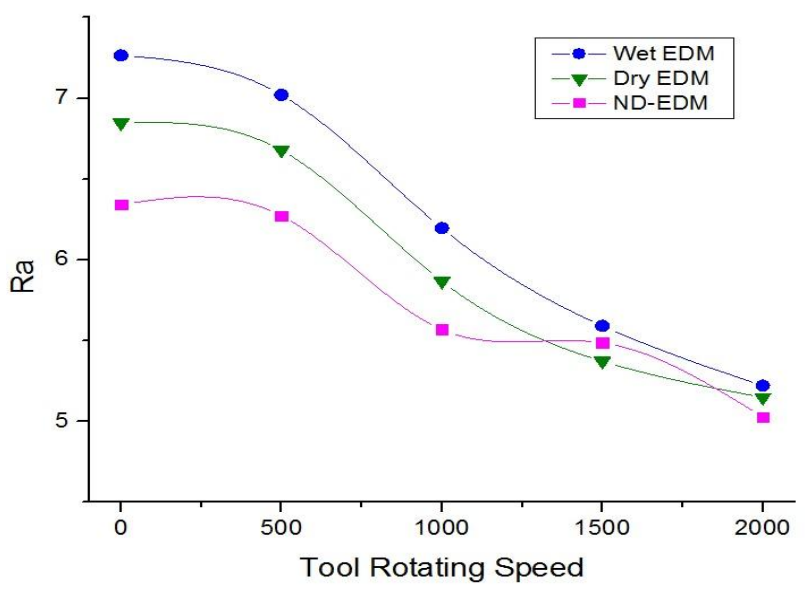

Figure 3: Effect of Tool Rotating Speed vs Ra

\section{CONCLUSION}

The effect of tool rotating speed to the responses viz. MRR, TWR and Ra for EDM processes viz. Wet EDM, Dry EDM and ND-EDM was carried. It can be concluded that ND-EDM process has maximum MRR, negligible TWR and minimum surface roughness from the Wet EDM and Dry EDM process.

\section{ACKNOWLEDGMENT}

Authors like to acknowledge the National Institute of Technology Manipur for kind co-operation to conduct the experiments.

\section{REFERENCES}

[1] K.H. Ho, S.T. Newman, "State of the art electrical discharge machining (EDM)", International Journal of Machine Tools \& Manufacture, Vol. 43, Pp. 1287-1300, 2013.

[2] S. Singh, S. Maheshwari, P.C. Pandey, "Some investigations into the electric discharge machining of hardened tool steel using different electrode materials", Journal of Materials Processing Technology, Vol. 149, Pp. 272-277, 2004.

[3] C.J. Luis, I. Puertas, G. Villa, "Material removal rate and electrode wear study on the EDM of silicon carbide", Journal of Materials Processing Technology, Vol. 164-165, Pp. 889-896, 2005.

[4] J. Marafona, J.A.G. Chousal, "A finite element model of EDM based on the Joule effect", International Journal of Machine Tools \& Manufacture, Vol. 46, Pp. 1-8, 2005.

[5] S. K. Saha and S. K. Choudhury, "Experimental investigation and empirical modeling of the dry electric discharge machining process", International J. of Machine Tools \& Manufacture, Vol. 49, Pp. 297-308, 2009.

[6] C. C. Kao, J. Tao, S. W. Lee and A. J. Shih, "Dry wire electrical discharge machining of thin workpiece", Trans. NAMRI/SME, Vol. 34, Pp. 253-260, 2006.

[7] T. Tambura, K. Isuzugawa, I. Fujita, "Development of EDM in the Mist", Proceedings of 9th International Symposium for ElectroMachining (ISEM-9) Nagoya, Pp. 313-316, 1989.

[8] C. C. Kao, J. Tao and A. J. Shih, "Near-dry electrical discharge machining", International J. of Machine Tools \& Manufacture, Vol. 47, Pp. 2273-228, 2007. 